\title{
Generalized hybrid integral Meler-Fok transform of the second type and its applications
}

\author{
I. M. Konet
}




\title{
GENERALIZED HYBRID INTEGRAL MELER-FOK TRANSFORM OF THE SECOND TYPE AND ITS APPLICATIONS
}

\author{
I. M. KONET
}

Received 29 September, 2006

\begin{abstract}
By the method of delta-like sequence, a generalized integral transformation of MelerFok type one the segment $\left[R_{0}, R\right]$ with $n$ contact points is obtained. We consider main examples of application of the transform for the solution of singular boundary value problems of mathematical physics of non-isotopic solids.
\end{abstract}

1991 Mathematics Subject Classification: 34D20

Keywords: generalized Legendres differential operator, integral transform, boundary value problems of mathematical physics

\section{INTRODUCTION}

In solving linear boundary and mixed problems of mathematical physics of homogeneous environments in the spherical system of coordinates by the method of separation of variables, the equations with the differential Legendres operator

$$
\Lambda_{m}=\frac{d^{2}}{d r^{2}}+\operatorname{cth} r \frac{d}{d r}+\frac{1}{4}-\frac{m^{2}}{\operatorname{sh}^{2} r}, \quad m \geq 0
$$

arise. The direct

$$
F_{0}[f(r)]=\int_{0}^{\infty} f(r) P_{-\frac{1}{2}+i \lambda}(\operatorname{ch} r) \operatorname{sh} r d r \equiv \tilde{f}(\lambda)
$$

and inverse

$$
F_{0}^{-1}[\tilde{f}(\lambda)]=\int_{0}^{\infty} \tilde{f}(\lambda) P_{-\frac{1}{2}+i \lambda}(\operatorname{ch} r) \lambda \operatorname{th}(\pi \lambda) d \lambda \equiv f(r)
$$

integral transforms generated in the polar axis $r \geq 0$ by the Legendres differential operator

$$
\Lambda_{0}=\frac{d^{2}}{d r^{2}}+\operatorname{cth} r \frac{d}{d r}+\frac{1}{4}
$$

were first obtained in 1861 by F. G. Meler and strictly substantiated by V. A. Fok [4] and M. M. Lebedev [12]. These transforms are efficiently used for solving axis-symmetrical problems of the theory of potential in the domains formed by two 
spheres that intersect and in the domains limited by the surfaces of hyperboloids of rotation and toroidal surfaces.

In the case of absence of axial symmetry the generalized integral transforms of Meler-Fok are used [14]:

$$
\begin{aligned}
F_{m}[f(r)] & =\int_{0}^{\infty} f(r) P_{-\frac{1}{2}+i \lambda}^{m}(\operatorname{ch} r) \operatorname{sh} r d r \equiv \tilde{f}(\lambda), \\
F_{m}^{-1}[\tilde{f}(\lambda)] & =(-1)^{m} \int_{0}^{\infty} \tilde{f}(\lambda) P_{-\frac{1}{2}+i \lambda}^{-m}(\operatorname{ch} r) \lambda \operatorname{th}(\pi \lambda) d \lambda \equiv f(r) ;
\end{aligned}
$$

they are generated by a differential operator $\Lambda_{m}(m=1,2,3, \ldots)$ on the polar axis $r \geq 0$. The integral transforms of Meler-Fok on the polar axis $r \geq R_{0}>0$ were obtained in the papers $[1,15,16]$.

A natural generalization of the differential operator $(1.1)$ is $[3,17]$

$$
\Lambda_{(\mu)}=\frac{d^{2}}{d r^{2}}+\operatorname{cth} r \frac{d}{d r}+\frac{1}{4}+\frac{1}{2}\left(\frac{\mu_{1}^{2}}{1-\operatorname{ch} r}+\frac{\mu_{2}^{2}}{1+\operatorname{ch} r}\right),
$$

where $(\mu)=\left(\mu_{1} ; \mu_{2}\right) ; \mu_{1} \geq \mu_{2}>0$. Operator (1.7) will be called the generalized Legendres differential operator. It is obvious that at $\mu_{1}=\mu_{2}=m$ an operator (1.7) coincides with operator (1.1).

The integral transforms of Meler-Fok type generated on the polar axis $r \geq 0$, $r \geq R_{0}>0$ and the polar segments $[0, R]$ and $\left[R_{0}, R\right]$ by the generalized Legendres differential operator (1.7) are obtained in [8-10]. Proper hybrid integral transforms, generated in these axes with one, two and $n$ contact points by a hybrid Legendres differential operator are considered in [5-7]. In this paper, by using the method of a delta-like sequence [13], limited hybrid integral transforms of Meler-Fok type are constructed on the segment $\left[R_{0}, R\right]$ with $n$ contact points. The transforms obtained are applied to the solving of some singular boundary problems of mathematical physics.

\section{BASIC PART}

Let us construct the limited integral transform generated in the set

$$
I_{n}=\left\{r: r \in \bigcup_{k=1}^{n+1}\left(R_{k-1}, R_{k}\right) ; R_{0}>0, R_{n+1} \equiv R<\infty\right\}
$$

by the generalized hybrid Legendres differential operator

$$
L_{(\mu)}=\sum_{k}^{n+1} \theta\left(r-R_{k-1} \theta\right)\left(R_{k}-r\right) a_{k}^{2} \Lambda_{(\mu)_{k}},
$$

where $(\mu)=\left((\mu)_{1},(\mu)_{2}, \ldots,(\mu)_{n+1}\right),(\mu)_{k}=\left(\mu_{1 k}, \mu_{2 k}\right)$, and $\theta(x)$ is the Heavyside function. 
For the domain of definition of the operator $L_{(\mu)}$ we take the set $G$ of vectorfunctions $g=\left(g_{1}, g_{2}, \ldots, g_{n}, g_{n+1}\right)$ which have the following properties:

(1) The vector-function

$$
f(r)=\left(\Lambda_{(\mu)_{1}}\left[g_{1}(r)\right] ; \Lambda_{(\mu)_{2}}\left[g_{2}(r)\right] ; \ldots ; \Lambda_{(\mu)_{n+1}}\left[g_{n+1}(r)\right]\right)
$$

is continuous on the set $I_{n}$;

(2) The components $g_{j}(r)$ of the vector-function $g(r)$ satisfy the conjugate conditions

$$
\left.\left[\left(\alpha_{j 1}^{k} \frac{d}{d r}+\beta_{j 1}^{k}\right) g_{k}(r)-\left(\alpha_{j 2}^{k} \frac{d}{d r}+\beta_{j 2}^{k}\right) g_{k+1}(r)\right]\right|_{r=R_{k}}=0,
$$

$j=1,2, k=\overline{1, n}$;

(3) The boundary conditions

$$
\begin{array}{r}
\left.\left(\alpha_{11}^{0} \frac{d}{d r}+\beta_{11}^{0}\right) g_{1}(r)\right|_{r=R_{0}}=0 \quad\left(\text { or } g_{0} \neq 0\right), \\
\left.\left(\alpha_{22}^{n+1} \frac{d}{d r}+\beta_{22}^{n+1}\right) g_{n+1}(r)\right|_{r=R_{n+1}}=0 \quad\left(\text { or } g_{R} \neq 0\right) .
\end{array}
$$

are satisfied.

We suppose that $c_{1 k} c_{2 k}>0, \alpha_{11}^{0} \leq 0, \beta_{11}^{0} \geq 0, \alpha_{22}^{n+1} \geq 0, \beta_{22}^{n+1} \geq 0$,

$$
\left|\alpha_{11}^{0}\right|+\beta_{11}^{0} \neq 0, \quad \alpha_{22}^{n+1}+\beta_{22}^{n+1} \neq 0, \quad c_{j k}=\alpha_{2 j}^{k} \beta_{1 j}^{k}-\alpha_{1 j}^{k} \beta_{2 j}^{k} .
$$

Let us define the numbers

$$
\sigma_{k}=\frac{1}{a_{k}^{2}} \prod_{j=k}^{n} \frac{c_{1 j}}{c_{2 j}}, \quad k=\overline{1, n}, \quad \sigma_{n}=\frac{1}{a_{n}^{2}} \frac{c_{1 n}}{c_{2 n}}, \quad \sigma_{n+1}=\frac{1}{a_{n+1}^{2}},
$$

and the gravimetric function

$$
\sigma(r)=\left(\sum_{k=1}^{n+1} \theta\left(r-R_{k-1}\right) \theta\left(R_{k}-r\right) \sigma_{k}\right) \operatorname{sh} r
$$

The scalar product of the elements of set $G$ will be defined by a formula

$$
(u, v)=\int_{R_{0}}^{R} u(r) v(r) \sigma(r) d r \equiv \sum_{k=1}^{n+1} \int_{R_{k-1}}^{R_{k}} u_{k}(r) v_{k}(r) \sigma_{k} \operatorname{sh} r d r
$$

where $u \in G, v \in G$. 
Theorem 1. The components of the vector-functions $u$ and $v$ from the domain of definition of operator $L_{(\mu)}$ satisfy the basic identity

$$
\begin{aligned}
u_{k}^{\prime}\left(R_{k}\right) v_{k}\left(R_{k}\right)- & u_{k}\left(R_{k}\right) v_{k}^{\prime}\left(R_{k}\right) \\
& =\frac{c_{2 k}}{c_{1 k}}\left(u_{k+1}^{\prime}\left(R_{k}\right) v_{k+1}\left(R_{k}\right)-u_{k+1}\left(R_{k}\right) v_{k+1}^{\prime}\left(R_{k}\right)\right) .
\end{aligned}
$$

Proof. Let us define the numbers

$$
\begin{array}{llrl}
c_{11}^{k} & =\alpha_{11}^{k} \alpha_{22}^{k}-\alpha_{21}^{k} \alpha_{12}^{k}, & c_{12}^{k} & =\alpha_{11}^{k} \beta_{22}^{k}-\alpha_{21}^{k} \beta_{12}^{k}, \\
c_{21}^{k} & =\beta_{11}^{k} \alpha_{22}^{k}-\beta_{21}^{k} \alpha_{12}^{k}, & c_{22}^{k} & =\beta_{11}^{k} \beta_{22}^{k}-\beta_{21}^{k} \beta_{12}^{k}
\end{array}
$$

for $k=\overline{1, n}$. From the conjugate conditions

$$
\begin{aligned}
& \alpha_{11}^{k} u_{k}^{\prime}\left(R_{k}\right)+\beta_{11}^{k} u_{k}\left(R_{k}\right)=\alpha_{12}^{k} u_{k+1}^{\prime}\left(R_{k}\right)+\beta_{12}^{k} u_{k+1}\left(R_{k}\right), \\
& \alpha_{21}^{k} u_{k}^{\prime}\left(R_{k}\right)+\beta_{21}^{k} u_{k}\left(R_{k}\right)=\alpha_{22}^{k} u_{k+1}^{\prime}\left(R_{k}\right)+\beta_{22}^{k} u_{k+1}\left(R_{k}\right),
\end{aligned}
$$

by Cramer rule [11] we find the relations

$$
\left.\begin{array}{l}
u_{k}^{\prime}\left(R_{k}\right)=c_{1 k}^{-1}\left[a_{21}^{k} u_{k+1}^{\prime}\left(R_{k}\right)+a_{22}^{k} u_{k+1}\left(R_{k}\right)\right], \\
u_{k}\left(R_{k}\right)=-c_{1 k}^{-1}\left[a_{11}^{k} u_{k+1}^{\prime}\left(R_{k}\right)+a_{12}^{k} u_{k+1}\left(R_{k}\right)\right] .
\end{array}\right\}
$$

Like for $v(r) \in G$, we have

$$
\left.\begin{array}{l}
v_{k}^{\prime}\left(R_{k}\right)=c_{1 k}^{-1}\left[a_{21}^{k} v_{k+1}^{\prime}\left(R_{k}\right)+a_{22}^{k} v_{k+1}\left(R_{k}\right)\right], \\
v_{k}\left(R_{k}\right)=-c_{1 k}^{-1}\left[a_{11}^{k} v_{k+1}^{\prime}\left(R_{k}\right)+a_{12}^{k} v_{k+1}\left(R_{k}\right)\right] .
\end{array}\right\}
$$

On the basis of equalities (2.3), (2.4) we find

$$
\begin{aligned}
u_{k}^{\prime}\left(R_{k}\right) v_{k}\left(R_{k}\right)-u_{k}\left(R_{k}\right) & v_{k}^{\prime}\left(R_{k}\right)=\frac{1}{c_{1 k}^{2}}\left(a_{11}^{k} a_{22}^{k}-a_{12}^{k} a_{21}^{k}\right) \\
& \times\left(u_{k+1}^{\prime}\left(R_{k}\right) v_{k+1}\left(R_{k}\right)-u_{k+1}\left(R_{k}\right) v_{k+1}^{\prime}\left(R_{k}\right)\right) .
\end{aligned}
$$

Taking into account the inequality $a_{11}^{k} a_{22}^{k}-a_{12}^{k} a_{21}^{k}=c_{1 k} c_{2 k}>0$, we obtain the basic identity (2.2). The theorem is proved.

Theorem 2. The generalized Legendres differential operator $L_{(\mu)}$ defined by equality (2.1) is selfconjugate.

Proof. For any $u(r) \in G$ and $v(r) \in G$ we have directly

$$
\left(L_{(\mu)}[u], v\right)=\sum_{k=1}^{n+1} \int_{R_{k-1}}^{R_{k}} a_{k}^{2} \Lambda_{(\mu)_{k}}\left[u_{k}\right] v_{k}(r) \sigma_{k} \operatorname{sh} r d r .
$$


Let us integrate by parts twice. We get

$$
\begin{aligned}
\sum_{k=1}^{n+1} \int_{R_{k-1}}^{R_{k}} a_{k}^{2} \Lambda_{(\mu)_{k}}\left[u_{k}\right] v_{k}(r) \sigma_{k} \operatorname{sh} r d r \\
=\sum_{k=1}^{n+1} a_{k}^{2}\left\{\left.\left[u_{k}^{\prime}(r) v_{k}(r)-u_{k}(r) v_{k}^{\prime}(r)\right] \sigma_{k} \operatorname{sh} r\right|_{R_{k-1}} ^{R_{k}}\right. \\
\left.\quad+\int_{R_{k-1}}^{R_{k}} a_{k}^{2} u_{k}(r) \Lambda_{(\mu)_{k}}\left[v_{k}(r)\right] \sigma_{k} \operatorname{sh} r d r\right\} \\
=\left(u(r), \Lambda_{(\mu)}[v(r)]\right) \\
\quad+\sum_{k=1}^{n}\left\{\sigma_{k} \operatorname{sh} R_{k}\left[u_{k}^{\prime}\left(R_{k}\right) v_{k}\left(R_{k}\right)-u_{k}\left(R_{k}\right) v_{k}^{\prime}\left(R_{k}\right)\right] a_{k}^{2}\right. \\
\left.\quad-a_{k+1}^{2} \sigma_{k+1} \operatorname{sh} R_{k}\left[u_{k+1}^{\prime}\left(R_{k}\right) v_{k+1}\left(R_{k}\right)-u_{k+1}\left(R_{k}\right) v_{k+1}^{\prime}\left(R_{k}\right)\right]\right\} \\
\quad+\sigma_{n+1} a_{n+1}^{2} \operatorname{sh} R_{k+1}\left[u_{n+1}^{\prime}(r) v_{n+1}(r)-u_{n+1}(r) v_{n+1}^{\prime}(r)\right] \\
\quad-\sigma_{1} a_{1}^{2} \operatorname{sh} R_{0}\left[u_{1}^{\prime}\left(R_{0}\right) v_{1}\left(R_{0}\right)-u_{1}\left(R_{0}\right) v_{1}^{\prime}\left(R_{0}\right)\right] .
\end{aligned}
$$

Due to the basic identity (2.2) and the structure of $\sigma_{k}$ we have

$$
\begin{aligned}
a_{k}^{2} \sigma_{k} \operatorname{sh} R_{k} & {\left[u_{k}^{\prime}\left(R_{k}\right) v_{k}\left(R_{k}\right)-u_{k}\left(R_{k}\right) v_{k}^{\prime}\left(R_{k}\right)\right] } \\
& =a_{k}^{2} \sigma_{k} \operatorname{sh} R_{k} \frac{c_{2 k}}{c_{1 k}}\left[u_{k+1}^{\prime}\left(R_{k}\right) v_{k+1}\left(R_{k}\right)-u_{k+1}\left(R_{k}\right) v_{k+1}^{\prime}\left(R_{k}\right)\right] \\
& =a_{k+1}^{2} \sigma_{k+1} \operatorname{sh} R_{k}\left[u_{k+1}^{\prime}\left(R_{k}\right) v_{k+1}\left(R_{k}\right)-u_{k+1}\left(R_{k}\right) v_{k+1}^{\prime}\left(R_{k}\right)\right] .
\end{aligned}
$$

Consequently, expression in the curly braces equals to zero. If $\alpha_{11}^{0} \neq 0$, as a result of the boundary condition in point $r=R_{0}$, we have

$$
\begin{aligned}
& \left.\left(\frac{d u_{1}}{d r} v_{1}-u_{1} \frac{d v_{1}}{d r}\right)\right|_{r=R_{0}} \\
& =\left.\left[\frac{1}{\alpha_{11}^{0}}\left(\alpha_{11}^{0} \frac{d u_{1}}{d r}+\beta_{11}^{0} u_{1}\right)-\frac{\beta_{11}^{0}}{\alpha_{11}^{0}} u_{1} v_{1}-u_{1} \frac{d v_{1}}{d r}\right]\right|_{r=R_{0}} \\
& =\left.\frac{v_{1}\left(R_{0}\right)}{\alpha_{11}^{0}}\left(\alpha_{11}^{0} \frac{d u_{1}}{d r}+\beta_{11}^{0} u_{1}\right)\right|_{r=R_{0}}-\left.\frac{u_{1}\left(R_{0}\right)}{\alpha_{11}^{0}}\left(\alpha_{11}^{0} \frac{d v_{1}}{d r}+\beta_{11}^{0} v_{1}\right)\right|_{r=R_{0}} \\
& =\left(\alpha_{11}^{0}\right)^{-1}\left[v_{1}\left(R_{0}\right) \cdot 0-u_{1}\left(R_{0}\right) \cdot 0\right] \equiv 0 \text {. }
\end{aligned}
$$


If $\alpha_{22}^{n+1} \neq 0$, in view of the boundary condition at the point $r=R_{n+1}$, we get

$$
\begin{gathered}
\left.\left(\frac{d u_{n+1}}{d r} v_{n+1}-u_{n+1} \frac{d v_{n+1}}{d r}\right)\right|_{r=R_{n+1}} \\
=\alpha_{22}^{n+1}\left[\left.v_{n+1}\left(R_{n+1}\right)\left(\alpha_{22}^{n+1} \frac{d}{d r}+\beta_{22}^{n+1}\right) u_{n+1}(r)\right|_{r=R}\right. \\
\left.\left.u_{n+1}\left(R_{n+1}\right)\left(\alpha_{22}^{n+1} \frac{d}{d r}+\beta_{22}^{n+1}\right) v_{n+1}(r)\right|_{r=R_{n+1}}\right] \\
=\frac{v_{n+1}\left(R_{n+1}\right)}{\alpha_{22}^{n+1}} \cdot 0-\frac{u_{n+1}\left(R_{n+1}\right)}{\alpha_{22}^{n+1}} \cdot 0 \equiv 0 .
\end{gathered}
$$

Consequently, as a result of the boundary conditions, expressions in points $r=R_{0}$ and $r=R \equiv R_{n+1}$ equal a zero. Equality (2.5) gives

$$
\left(L_{(\mu)}[u], v\right)=\sum_{k=1}^{n+1} a_{k}^{2} \int_{R_{k-1}}^{R_{k}} \Lambda_{(\mu)_{k}}\left[u_{k}\right] v_{k}(r) \sigma_{k} \operatorname{sh} r d r=\left(u, L_{(\mu)}[v]\right) .
$$

The last equality means that the operator $L_{(\mu)}$ is selfconjugate. The theorem is proved.

As an operator $L_{(\mu)}$ in the set $I_{n}$ has not the special points and is selfconjugate, its spectrum is discrete and real [2].

Let us find eigenvalues and vector eigenfunctions of the GDLO $L_{(\mu)}$ defined by equality (2.1) as a solution of spectral Schturm-Liouville problem: to construct nontrivial limited in the set $I_{n}$ solution of the separate system of the generalized Legendres differential equations

$$
\left(\Lambda_{(\mu)_{j}}+b_{j}^{2}\right) V_{(\mu) ; j}(r, \beta)=0, \quad r \in\left(R_{j-1}, R_{j}\right), \quad j=\overline{1, n+1}
$$

under the boundary conditions

$$
\begin{array}{r}
\left.\left(\alpha_{11}^{0} \frac{d}{d r}+\beta_{11}^{0}\right) V_{(\mu) ; 1}(r, \beta)\right|_{r=R_{0}}=0 \\
\left.\left(\alpha_{22}^{n+1} \frac{d}{d r}+\beta_{22}^{n+1}\right) V_{(\mu) ; n+1}(r, \beta)\right|_{r=R_{n+1}}=0
\end{array}
$$

and the conjugate conditions

$$
\begin{aligned}
& {\left[\left(\alpha_{j 1}^{k} \frac{d}{d r}+\beta_{j 1}^{k}\right) V_{(\mu) ; k}(r, \beta)\right.} \\
& \left.\quad-\left(\alpha_{j 2}^{k} \frac{d}{d r}+\beta_{j 2}^{k}\right) V_{(\mu) ; k+1}(r, \beta)\right]\left.\right|_{r=R_{k}}=0, \quad j=1,2, k=\overline{1, n},
\end{aligned}
$$


where $b_{j}=a_{j}^{-1}\left(\beta^{2}+\gamma_{j}^{2}\right)^{1 / 2}, a_{j}>0, \gamma_{j}^{2} \geq 0, j=\overline{1, n+1}$, and $\beta$ is a spectral parameter.

The presence of the fundamental system of solutions [17] allows us to search for the solution of homogeneous boundary problem (2.6), (2.7), (2.8) according to the rule

$$
V_{(\mu) ; j}(r, \beta)=C_{j} A_{-1 / 2+i b_{j}}^{(\mu)_{j}}(\operatorname{ch} r)+D_{j} B_{-1 / 2+i b_{j}}^{(\mu)_{j}}(\operatorname{ch} r), \quad j=\overline{1, n+1} .
$$

The boundary conditions (2.7) and conjugate conditions (2.8) for the determination of the values $C_{j}$ and $D_{j}(j=\overline{1, n+1})$ give the following system of $(2 n+2)=$ $2(n+1)$ algebraic equations:

$$
\left.\begin{array}{c}
Y_{-1 / 2+i b_{1} ; 11}^{(\mu)_{1}, 01}\left(\operatorname{ch~} R_{0}\right) C_{1}+Y_{-1 / 2+i b_{1} ; 11}^{(\mu)_{1}, 02}\left(\operatorname{ch} R_{0}\right) D_{1}=0, \\
Y_{-1 / 2+i b_{j} ; m 1}^{(\mu)_{j}, j 1}\left(\operatorname{ch~} R_{j}\right) C_{j}+Y_{-1 / 2+i b_{j} ; m 1}^{(\mu)_{j}, j 2}\left(\operatorname{ch} R_{j}\right) D_{j} \\
-Y_{-1 / 2+i b_{j+1} ; m_{2}}^{(\mu)_{j+1}, j_{1}}\left(\operatorname{ch} R_{j}\right) C_{j+1} \\
\quad-Y_{-1 / 2+i b_{j+1} ; m_{2}}^{(\mu)_{j+1}, j_{2}}\left(\operatorname{ch} R_{j}\right) D_{j+1}=0, \quad j=\overline{1, n}, \\
Y_{-1 / 2+i b_{n+1} ; 22}^{(\mu)_{n+1} ; n+1,1}\left(\operatorname{ch~} R_{n+1}\right) C_{n+1} \\
+Y_{-1 / 2+i b_{n+1} ; 22}^{(\mu)_{n+1} ; n+1,2}\left(\operatorname{ch} R_{n+1}\right) D_{n+1}=0, \quad m=1,2 .
\end{array}\right\}
$$

Let us define the functions

$$
\begin{aligned}
& \omega_{(\mu)_{1} ; 1}^{(0)}(\beta)=Y_{-1 / 2+i b_{1} ; 11}^{(\mu)_{1} ; 01}\left(\operatorname{ch~} R_{0}\right), \\
& \omega_{(\mu)_{1} ; 2}^{(0)}(\beta)=Y_{-1 / 2+i b_{1} ; 11}^{(\mu)_{1} ; 02}\left(\operatorname{ch} R_{0}\right)
\end{aligned}
$$

and put

$$
\begin{aligned}
\psi_{\left((\mu)_{k} ;(\mu)_{k+1}\right) ; m j}^{k} & \left(\beta, \operatorname{ch} R_{k}, \operatorname{ch} R_{k+1}\right) \\
= & Y_{-1 / 2+i b_{k} ; 11}^{(\mu)_{k} ; k m}\left(\operatorname{ch} R_{k}\right) Y_{-1 / 2+i b_{k+1} ; 22}^{(\mu)_{k+1} ; k j}\left(\operatorname{ch} R_{k+1}\right) \\
& -Y_{-1 / 2+i b_{k} ; 21}^{(\mu)_{k} ; k m}\left(\operatorname{ch} R_{k}\right) Y_{-1 / 2+i b_{k+1} ; 12}^{(\mu)_{k+1} ; k j}\left(\operatorname{ch~} R_{k+1}\right), \quad k=\overline{1, n}
\end{aligned}
$$

and

$$
\begin{aligned}
& \omega_{(\mu)_{k+1} ; j}^{(k)}(\beta)=\omega_{(\mu)_{k} ; 2}^{(k-1)}(\beta) \psi_{\left((\mu)_{k} ;(\mu)_{k+1}\right) ; 1 j}^{k}\left(\beta, \operatorname{ch} R_{k}, \operatorname{ch} R_{k+1}\right) \\
& \quad-\omega_{(\mu)_{k} ; 1}^{(k-1)}(\beta) \psi_{\left((\mu)_{k} ;(\mu)_{k+1}\right) ; 2 j}^{k}\left(\beta, \operatorname{ch} R_{k}, \operatorname{ch} R_{k+1}\right), \quad j=1,2, k=\overline{1, n}
\end{aligned}
$$

where $(\mu)_{k}=\left((\mu)_{1},(\mu)_{2}, \ldots,(\mu)_{k}\right),(\mu)=\left((\mu)_{1},(\mu)_{2}, \ldots,(\mu)_{n},(\mu)_{n+1}\right)$. 
In order that the algebraic system (2.9) have a non-trivial solution, it is necessary and sufficient that its determinant be equal zero [11]:

$$
\begin{aligned}
\delta_{(\mu) ; n}(\beta) \equiv Y_{-1 / 2+i b_{n+1} ; 22}^{(\mu)_{n+1} ; n+1,1}(\operatorname{ch} r) \omega_{(\mu)_{n+1} ; 2}^{(n)}(\beta) & \\
& -Y_{-1 / 2+i b_{n+1} ; 22}^{(\mu)_{n+1} ; n+1,2}(\operatorname{ch} r) \omega_{(\mu)_{n+1} ; 1}^{(n)}(\beta)=0 .
\end{aligned}
$$

The transcendent equation (2.10) is the equation for determination of eigenvalues of the operator $L_{(\mu)}$.

Theorem 3 (On the discrete spectrum). The solutions $\beta_{m}$ of the transcendent equation (2.10) form a discrete spectrum: they are real, distinct, located symmetrically with respect to $\beta=0$, their moduli make a droningly growing number sequence with a unique maximum point $\beta=\infty$.

We will put in the system $(2.10) \beta=\beta_{m}\left(b_{j}\left(\beta_{m}\right) \equiv b_{j m}=a_{j}^{-1}\left(\beta_{m}^{2}+\gamma_{j}^{2}\right)^{1 / 2}\right)$ and we will cast aside the last equation as a result of linear dependence. If

$$
\begin{aligned}
V_{(\mu) ; 1}\left(r, \beta_{m}\right)=A_{0}\left(\omega_{(\mu)_{1} ; 2}^{(0)}\left(\beta_{m}\right) A_{-1 / 2+i b_{1 m}}^{(\mu)_{1}}(\operatorname{ch} r)\right. & \\
& \left.-\omega_{(\mu)_{1} ; 1}^{(0)}\left(\beta_{m}\right) B_{-1 / 2+i b_{1 m}}^{(\mu)_{1}}(\operatorname{ch} r)\right),
\end{aligned}
$$

where $A_{0}=A_{0}\left(\beta_{m}\right)$ is subject to determination, then equations of the system, except the first and last, form $n$ recurrent systems on two equations in each. The first equation of the system is satisfied. At

$$
A_{0} \equiv \Delta_{(\mu) ; n}\left(\beta_{m}\right)=\prod_{k=1}^{n} \frac{c_{2 k}}{\operatorname{sh} R_{k}} \frac{1}{S_{(\mu)_{k}}\left(b_{k m}\right)},
$$

as a result of solving the recurrent systems, we get the structure of the components of vector eigenfunction

$$
\begin{aligned}
V_{(\mu) ; 1}\left(r, \beta_{m}\right)= & \Delta_{(\mu) ; n}\left(\beta_{m}\right)\left(\omega_{(\mu)_{1} ; 2}^{(0)}\left(\beta_{m}\right) A_{-1 / 2+i b_{1 m}}^{(\mu)_{1}}(\operatorname{ch} r)\right. \\
& \left.-\omega_{(\mu)_{1} ; 1}^{(0)}\left(\beta_{m}\right) B_{-1 / 2+i b_{1 m}}^{(\mu)_{1}}(\operatorname{ch} r)\right), \\
V_{(\mu) ; k}\left(r, \beta_{m}\right)= & \left(\prod_{s=k}^{n} \frac{c_{2 s}}{\operatorname{sh} R_{s}} \frac{1}{S_{(\mu)_{s}}\left(b_{s m}\right)}\right)\left[\omega_{(\mu)_{k} ; 2}^{(k-1)}\left(\beta_{m}\right) A_{-1 / 2+i b_{k m}}^{(\mu)_{k}}(\operatorname{ch} r)\right. \\
& \left.-\omega_{(\mu)_{k} ; 1}^{(k-1)}\left(\beta_{m}\right) B_{-1 / 2+i b_{k m}}^{(\mu)_{k}}(\operatorname{ch} r)\right] ; \quad k=\overline{2, n} ; \\
V_{(\mu) ; n+1}\left(r, \beta_{m}\right)=\omega_{\left((\mu)_{n+1} ; 2\right.}^{(n)}\left(\beta_{m}\right) A_{-1 / 2+i b_{n+1, m}}^{(\mu)_{n+1}}(\operatorname{ch} r) & -\omega_{(\mu)_{n+1} ; 1}^{(n)}\left(\beta_{m}\right) B_{-1 / 2+i b_{n+1, m}}^{(\mu)_{n+1}}(\operatorname{ch} r) .
\end{aligned}
$$


To an eigenvalue $\beta_{m}$, one (spectral) vector eigenfunction corresponds,

$$
V_{(\mu)}\left(r, \beta_{m}\right)=\sum_{k=1}^{n+1} \theta\left(r-R_{k-1}\right) \theta\left(R_{k}-r\right) V_{(\mu) ; k}\left(r, \beta_{m}\right)
$$

with the square of the norm equal to

$$
\begin{aligned}
\left\|V_{(\mu)}\left(r, \beta_{m}\right)\right\|^{2}=\left(V_{(\mu)}\left(r, \beta_{m}\right), V_{(\mu)}\right. & \left.\left(r, \beta_{m}\right)\right) \\
& \equiv \sum_{k=1}^{n+1} \int_{R_{k-1}}^{R_{k}}\left[V_{(\mu) ; k}\left(r, \beta_{m}\right)\right]^{2} \sigma_{k} \operatorname{sh} r d r .
\end{aligned}
$$

Theorem 4 (On a discrete function). The system of the vector eigenfunctions $\left\{V_{(\mu)}\left(r, \beta_{m}\right)\right\}_{m=1}^{\infty}$ is orthogonal and complete in the set $I_{n}$.

Theorem 5 (A Steklov-type theorem). Any vector-function $g(r) \in G$ is represented by absolutely and uniformly convergent in every compact set $I_{n}^{*} \subset I_{n}$ by a Fourier series for the system $\left\{V_{(\mu)}\left(r, \beta_{m}\right)\right\}_{m=1}^{\infty}$ of the vector eigenfunctions of the operator $L_{(\mu)}$ :

$$
g(r)=\sum_{m=1}^{\infty} \int_{R_{0}}^{R_{n+1}} g(\rho) V_{(\mu)}\left(\rho, \beta_{m}\right) \sigma(\rho) d \rho \frac{V_{(\mu)}\left(r, \beta_{m}\right)}{\left\|V_{(\mu)}\left(r, \beta_{m}\right)\right\|^{2}} .
$$

The Fourier series (2.11) determines the direct $M_{(\mu) ; 4 n}$ and inverse $M_{(\mu) ; 4 n}^{-1}$ limited generalized integral Meler-Fok transform of the second type generated in the set $I_{n}$ by the GDLO $L_{(\mu)}$ :

$$
\begin{aligned}
M_{(\mu) ; 4 n}[g(r)] & =\int_{R_{0}}^{R} g(r) V_{(\mu)}\left(r, \beta_{m}\right) \sigma(r) d r \equiv \tilde{g}_{m}=\sum_{k=1}^{n+1} \tilde{g}_{k m} \\
& =\sum_{k=1}^{n+1} \int_{R_{k-1}}^{R_{k}} g_{k}(r) V_{(\mu) ; k}\left(r, \beta_{m}\right) \sigma_{k} \operatorname{sh} r d r .
\end{aligned}
$$

and

$$
M_{(\mu) ; 4 n}^{-1}\left[\tilde{g}_{m}\right]=\sum_{m=1}^{\infty} \tilde{g}_{m} \frac{V_{(\mu)}\left(r, \beta_{m}\right)}{\left\|V_{(\mu)}\left(r, \beta_{m}\right)\right\|^{2}} \equiv g(r) .
$$

Theorem 6 (On the basic identity). If the vector-function $g \in G$, and the components $g_{k}$ of the vector-function $g$ satisfy innhomogeneous boundary conditions and the innhomogeneous conjugate conditions, then the basic identity for the integral 
transform of the GDLO $L_{(\mu)}$ determined by equality (2.1) is true:

$$
\begin{gathered}
M_{(\mu) ; 4 n}\left[L_{(\mu)}(g)\right]=-\beta_{m}^{2} \tilde{g}_{m}+\sigma_{n+1} a_{n+1}^{2} \\
\quad+\left(\alpha_{22}^{n+1}\right)^{-1} \operatorname{sh} R V_{(\mu) ; n+1}\left(R_{n+1}, \beta_{m}\right) g_{R} \\
-a_{1}^{2} \sigma_{1}\left(\alpha_{11}^{0}\right)^{-1} \operatorname{sh} R_{0} V_{(\mu) ; 1}\left(R_{0}, \beta_{m}\right) g_{0} \\
\quad-\sum_{k=1}^{n+1} \gamma_{k}^{2} \tilde{g}_{k m} \\
+\sum_{k=1}^{n} a_{k}^{2} \sigma_{k}\left(c_{1 k}\right)^{-1} \operatorname{sh} R_{k}\left(Z_{(\mu) ; 12}^{k}\left(\beta_{m}\right) \omega_{2 k}\right. \\
\left.\quad-Z_{(\mu) ; 22}^{k}\left(\beta_{m}\right) \omega_{1 k}\right) .
\end{gathered}
$$

The proof of Theorems $2-6$ repeats the logical scheme of proof of the respective theorems in [7].

\section{APPLICATIONS}

The presence of the basic identity (2.14) allows us to apply the integral transforms inculcated by formulas (2.12) and (2.13) for the construction of the exact analytical solution of the proper singular problems of the mathematical physics of inhomogeneous structures.

\subsection{An example: a statics problem}

Let us consider the problem to construct, in the domain $D_{n}=\left\{(r, z): r \in I_{n}\right.$; $z \in(-\infty ;+\infty)\}$, the bounded solution of the separate elliptic system of equation with the generalized Legendres operator

$$
\left(\frac{\partial^{2}}{\partial z^{2}}+a_{j}^{2} \Lambda_{(\mu) j}-\chi_{j}^{2}\right) u_{j}(r, z)=-f_{j}(r, z), \quad j=\overline{1, n+1}
$$

under the boundary conditions

$$
\begin{array}{r}
\left.\left(\alpha_{11}^{0} \frac{\partial}{\partial r}+\beta_{11}^{0}\right) u_{1}\right|_{r=R_{0}}=g_{0}(z), \\
\left.\left(\alpha_{22}^{n+1} \frac{\partial}{\partial r}+\beta_{22}^{n+1}\right) u_{n+1}\right|_{r=R_{n+1}}=g_{R}(z)
\end{array}
$$


and the conjugate conditions

$$
\begin{aligned}
& \left(\left(\alpha_{j 1}^{0} \frac{\partial}{\partial r}+\beta_{j 1}^{k}\right) u_{k}(r, z)\right. \\
& \left.\quad-\left(\alpha_{j 2}^{n+1} \frac{\partial}{\partial r}+\beta_{j 2}^{n+1}\right) u_{k+1}(r, z)\right)\left.\right|_{r=R_{k}}=\omega_{j k}(z),
\end{aligned}
$$

where $j=1,2, k=\overline{1, n}$.

We rewrite system (3.1) in the matrix form

$$
\left(\begin{array}{c}
\left(\frac{\partial^{2}}{\partial z^{2}}+a_{1}^{2} \Lambda_{(\mu)_{1}}-\chi_{1}^{2}\right) u_{1}(r, z) \\
\left(\frac{\partial^{2}}{\partial z^{2}}+a_{2}^{2} \Lambda_{(\mu)_{2}}-\chi_{2}^{2}\right) u_{2}(r, z) \\
\vdots \\
\left(\frac{\partial^{2}}{\partial z^{2}}+a_{n+1}^{2} \Lambda_{(\mu)_{n+1}}-\chi_{n+1}^{2}\right) u_{n+1}(r, z)
\end{array}\right)=-\left(\begin{array}{c}
f_{1}(r, z) \\
f_{2}(r, z) \\
\vdots \\
f_{n+1}(r, z)
\end{array}\right) .
$$

The integral operator $M_{(\mu) ; 4 n}$, in accordance with (2.12), we represent as the operator matrix-row

$$
\begin{aligned}
M_{(\mu) ; 4 n}[\cdots]=( & \int_{R_{0}}^{R_{1}} \cdots V_{(\mu) ; 1}\left(r, \beta_{m}\right) \sigma_{1} \operatorname{sh} r d r, \\
& \int_{R_{1}}^{R_{2}} \cdots V_{(\mu) ; 2}\left(r, \beta_{m}\right) \sigma_{2} \operatorname{sh} r d r, \\
& \cdots \cdots \cdots \\
& \int_{R_{n-1}}^{R_{n}} \cdots V_{(\mu) ; n}\left(r, \beta_{m}\right) \sigma_{n} \operatorname{sh} r d r \\
& \left.\int_{R_{n}}^{R_{n+1}} \cdots V_{(\mu) ; n+1}\left(r, \beta_{m}\right) \sigma_{n+1} \operatorname{sh} r d r\right) .
\end{aligned}
$$

Let us assume that $\chi_{1}^{2}=\max _{1 \leq j \leq n+1}\left\{\chi_{j}^{2}\right\}$ (in the contrary case, it is possible to renumber these values). Let us put $\gamma_{j}^{2}=\chi_{1}^{2}-\chi_{j}^{2} \geq 0$ for $j=\overline{1, n+1}$ and apply, by the rule of multiplication of matrices, the operator matrix-tow (3.4) to system (3.1). As a result of identity (2.14), we get a boundary problem on the structure of bounded solutions of the second-order ordinary differential equation with constant coefficients in the set $I_{1}=\{z: z \in(-\infty,+\infty)\}$

$$
\left(\frac{d^{2}}{d z^{2}}-q_{m}^{2}\right) \tilde{u}_{m}(z)=-\tilde{F}_{m}(z)
$$


where $q_{m}=\left(\beta_{m}^{2}+\chi_{1}^{2}\right)^{1 / 2}$ and

$$
\begin{aligned}
& \tilde{F}_{m}(z)=\tilde{f}_{m}(z)+\left(a_{22}^{n+1}\right)^{-1} \operatorname{sh} R_{n+1} V_{(\mu) ; n+1}\left(R, \beta_{m}\right) g_{R}(z) \\
&-\left(\alpha_{11}^{0}\right)^{-1} \operatorname{sh} R_{0} a_{1}^{2} \sigma_{1} V_{(\mu) ; 1}\left(R_{0}, \beta_{m}\right) g_{0}(z) \\
&+\sum_{k=1}^{n} a_{k}^{2} \sigma_{k} \frac{\operatorname{sh} R_{k}}{c_{1 k}}\left(Z_{(\mu) ; 12}^{k}\left(\beta_{m}\right) \omega_{2 k}(z)-Z_{(\mu) ; 22}^{k}\left(\beta_{(\mu)}\right) \omega_{1 k}(z)\right) .
\end{aligned}
$$

It is checked directly that the following function is the unique bounded solution of equation (3.5):

$$
\tilde{u}_{m}(z)=\int_{-\infty}^{\infty} \frac{1}{2 q_{m}} e^{-q_{m}|z-\zeta|} \tilde{F}_{m}(\zeta) d \zeta .
$$

The operator $M_{(\mu) ; 4 n}^{-1}$, in accordance with the rule (2.13), as inverse to (2.12), we will represent as the operator matrix-column

$$
M_{(\mu) ; 4 n}^{-1}[\cdots]=\left(\begin{array}{c}
\sum_{m=1}^{\infty} \cdots \frac{V_{(\mu) ; 1}\left(r, \beta_{m}\right)}{\left\|V_{(\mu)}\left(r, \beta_{m}\right)\right\|^{2}} \\
\sum_{m=1}^{\infty} \cdots \frac{V_{(\mu) ; 2}\left(r, \beta_{m}\right)}{\left\|V_{(\mu)}\left(r, \beta_{m}\right)\right\|^{2}} \\
\ldots \ldots \ldots \ldots \cdots \cdots \cdots \cdots \\
\sum_{m=1}^{\infty} \cdots \frac{V_{(\mu) ; n+1}\left(r, \beta_{m}\right)}{\left\|V_{(\mu)}\left(r, \beta_{m}\right)\right\|^{2}}
\end{array}\right) .
$$

We will apply by rule of multiplication of matrices the operator matrix-column to the matrix-element $\left[\tilde{u}_{m}(z)\right]$, where the function $\tilde{u}_{m}(z)$ is defined by formula (3.6). As a result of elementary transformations, we get the unique bounded solution of the elliptic problem (3.1), (3.2), (3.3):

$$
\begin{aligned}
u_{j}(r, z) & =\sum_{k=1}^{n+1} \int_{-\infty}^{\infty} \int_{R_{k-1}}^{R_{k}} E_{(\mu) ; j k}(r, \rho, z, \zeta) f_{k}(\rho, \zeta) \sigma_{k} \operatorname{sh} \rho d \rho d \zeta \\
& +\int_{-\infty}^{\infty}\left(W_{(\mu) ; 1 j}(r, z, \zeta) g_{0}(\zeta)+W_{(\mu) ; n+1, j}(r, z, \zeta) g_{R}(\zeta)\right) d \zeta \\
& +\sum_{k=1}^{n} \int_{-\infty}^{\infty}\left(R_{(\mu) ; 12}^{j k}(r, z, \zeta) \omega_{2 k}(\zeta)-R_{(\mu) ; 22}^{j k}(r, z, \zeta) \omega_{1 k}(\zeta)\right) d \zeta
\end{aligned}
$$

for $j=\overline{1, n+1}$.

Here, the principal solutions appear: (1) the influence functions generated by the inhomogeneity of system (3.1):

$$
E_{(\mu) ; j k}(r, \rho, z, \zeta)=\sum_{m=1}^{\infty} \frac{1}{2 q_{m}} e^{-q_{m}|z-\zeta|} \frac{V_{(\mu) ; j}\left(r, \beta_{m}\right) V_{(\mu) ; k}\left(\rho, \beta_{m}\right)}{\left\|V_{(\mu)}\left(r, \beta_{m}\right)\right\|^{2}},
$$

where $j, k=\overline{1, n+1}$; 
(2) Green's functions generated by boundary condition at $r=R_{0}$ :

$W_{(\mu) ; 1 k}(r, z, \zeta)=-a_{1}^{2} \sigma_{1} \operatorname{sh} R_{0} \sum_{m=1}^{\infty} \frac{e^{-q_{m}|z-\zeta|}}{2 q_{m}} \frac{V_{(\mu) ; 1}\left(R_{0}, \beta_{m}\right) V_{(\mu) ; j}\left(R_{0}, \beta_{m}\right)}{\alpha_{11}^{0}\left\|V_{(\mu)}\left(r, \beta_{m}\right)\right\|^{2}} ;$

(3) Green's functions generated by boundary condition at $r=R_{n+1} \equiv R$ :

$$
W_{(\mu) ; n+1, j}(r, z, \zeta)=\frac{\operatorname{sh} R}{\alpha_{22}^{n+1}} \sum_{m=1}^{\infty} \frac{e^{-q_{m}|z-\zeta|}}{2 q_{m}} \frac{V_{(\mu) ; j}\left(r, \beta_{m}\right) V_{(\mu) ; n+1}\left(R, \beta_{m}\right)}{\left\|V_{(\mu)}\left(r, \beta_{m}\right)\right\|^{2}},
$$

where $j=\overline{1, n+1}$;

(4) Green's functions generated by inhomogeneity of the conjugate conditions:

$$
R_{(\mu) ; s 2}^{j k}(r, z, \zeta)=\sum_{m=1}^{\infty} \frac{e^{-q_{m}|z-\zeta|}}{2 q_{m}} a_{k}^{2} \sigma_{k} \frac{\operatorname{sh} R_{k}}{c_{1 k}} Z_{(\mu) ; s 2}^{k}\left(\beta_{m}\right) \frac{V_{(\mu) ; j}\left(r, \beta_{m}\right)}{\left\|V_{(\mu)}\left(r, \beta_{m}\right)\right\|^{2}},
$$

where $s=1,2, k=\overline{1, n}$.

The vector-function $u(r, z)=\left\{u_{1}(r, z), u_{2}(r, z), \ldots, u_{n}(r, z), u_{n+1}(r, z)\right\}$ determines the integral image of the unique analytical solution of the given elliptic boundary problem.

\subsection{An example: a quasi-statics problem}

Let us construct in the domain $D_{n}^{+}=\left\{(t, r): t \in(0, \infty), r \in I_{n}\right\}$ the bounded solution of the separate system of equation of parabolic type with the generalized Legendres operator

$$
\frac{\partial u}{\partial t}+\chi_{j}^{2} u_{j}-a_{j}^{2} \Lambda_{(\mu) j}\left(u_{j}\right)=f_{j}(t, r), \quad j=\overline{1, n+1}
$$

with the initial conditions

$$
\left.u_{j}(t, r)\right|_{t=0}=g_{j}(r), r \in\left(R_{j-1}, R_{j}\right), \quad j=\overline{1, n+1},
$$

the boundary conditions

$$
\left.\begin{array}{r}
\left.\left(\alpha_{11}^{0} \frac{\partial}{\partial r}+\beta_{11}^{0}\right) u_{1}\right|_{r=R_{0}}=g_{0}(t), \\
\left.\left(\alpha_{22}^{n+1} \frac{\partial}{\partial r}+\beta_{22}^{n+1}\right) u_{n+1}\right|_{r=R_{n+1}}=g_{R}(t),
\end{array}\right\}
$$

and the conjugate conditions

$$
\begin{aligned}
&\left.\left(\left(\alpha_{j 1}^{k} \frac{\partial}{\partial r}+\beta_{j 1}^{k}\right) u_{k}-\left(\alpha_{j 2}^{k} \frac{\partial}{\partial r}+\beta_{j 2}^{k}\right) u_{k+1}\right)\right|_{r=R_{k}}=\omega_{j k}(t), \\
& j=1,2, k=\overline{1, n} .
\end{aligned}
$$


We assume that the compatibility conditions hold:

$$
\begin{array}{r}
\left.\left(\alpha_{11}^{0} \frac{d}{d r}+\beta_{11}^{0}\right) g_{1}\right|_{r=R_{0}}=g_{0}(0), \\
\left.\left(\alpha_{22}^{n+1} \frac{d}{d r}+\beta_{22}^{n+1}\right) g_{n+1}\right|_{r=R_{n+1}}=g_{R}(0),
\end{array}
$$

and

$$
\left.\left[\left(\alpha_{j 1}^{k} \frac{d}{d r}+\beta_{j 1}^{k}\right) g_{k}-\left(\alpha_{j 2}^{k} \frac{d}{d r}+\beta_{j 2}^{k}\right) g_{k+1}\right]\right|_{r=R_{k}}=\omega_{j k}(0)
$$

for $j=1,2, k=\overline{1, n}$.

We rewrite system (3.8) in the matrix form

$$
\left(\begin{array}{c}
\left(\frac{\partial}{\partial t}+\chi_{1}^{2}-a_{1}^{2} \Lambda_{\left.(\mu)_{1}\right)}\right) u_{1}(t, r) \\
\left(\frac{\partial}{\partial t}+\chi_{2}^{2}-a_{2}^{2} \Lambda_{(\mu)_{2}}\right) u_{2}(t, r) \\
\vdots \\
\left(\frac{\partial}{\partial t}+\chi_{n+1}^{2}-a_{n+1}^{2} \Lambda_{(\mu)_{n+1}}\right) u_{n+1}
\end{array}\right)=-\left(\begin{array}{c}
f_{1}(t, r) \\
f_{2}(t, r) \\
\vdots \\
f_{n+1}(t, r)
\end{array}\right)
$$

and, similarly, the initial conditions (3.9) in the form

$$
\left.\left(\begin{array}{c}
u_{1}(t, r) \\
u_{2}(t, r) \\
\vdots \\
u_{n+1}(t, r)
\end{array}\right)\right|_{t=0}=\left(\begin{array}{c}
g_{1}(r) \\
g_{2}(r) \\
\vdots \\
g_{n+1}(r)
\end{array}\right) .
$$

Supposing that $\chi_{1}^{2}=\max \left\{\chi_{1}^{2}, \chi_{2}^{2}, \ldots, \chi_{n}^{2}, \chi_{n+1}^{2}\right\}$, we will apply to problem (3.12), (3.13), by the rule of multiplication of matrices, the operator matrix-row (3.4). As a result of identity (2.14), we get the Cauchy problem

$$
\left(\frac{d}{d t}+q_{m}^{2}\right) \tilde{u}_{m}(t)=\tilde{F}_{m}(t),\left.\quad \tilde{u}_{m}\right|_{t=0}=g_{m} ;
$$

where $\tilde{F}_{m}(t)$ is as above.

The unique solution of the Cauchy problem (3.14) is the function

$$
\tilde{u}_{m}(t)=e^{-q_{m}^{2} t} g_{m}+\int_{0}^{t} e^{-q_{m}^{2}(t-\tau)} \tilde{F}_{m}(\tau) d \tau .
$$

Let us determine the principal solutions of the given parabolic problem: (1) the influence functions generated by the presence of the thermal sources

$$
H_{(\mu) ; j k}(t, r, \rho)=\sum_{m=1}^{\infty} e^{-q_{m}^{2} t} \frac{V_{(\mu) ; j}\left(r, \beta_{m}\right) V_{(\mu) ; k}\left(\rho, \beta_{m}\right)}{\left\|V_{(\mu)}\left(r, \beta_{m}\right)\right\|^{2}}, \quad j, k=\overline{1, n+1} ;
$$


(2) Green's functions generated by the boundary condition at $r=R_{0}$ :

$$
W_{(\mu) ; 1 j}(t, r)=-a_{1}^{2} \sigma_{1} \operatorname{sh} R_{0} \sum_{m=1}^{\infty} e^{-q_{m}^{2} t} \frac{V_{(\mu) ; 1}\left(R_{0}, \beta_{m}\right) V_{(\mu) ; j}\left(r, \beta_{m}\right)}{\alpha_{11}^{0}\left\|V_{(\mu)}\left(r, \beta_{m}\right)\right\|^{2}},
$$

$j=\overline{1, n+1}$

(3) Green's functions generated by the boundary condition at the point $r=R_{n+1}$ :

$$
W_{(\mu) ; n+1, j}(t, r)=\sum_{m=1}^{\infty} e^{-q_{m}^{2} t} \frac{\operatorname{sh} R}{\alpha_{22}^{n+1}} \frac{V_{(\mu) ; j}\left(r, \beta_{m}\right) V_{(\mu) ; n+1}\left(R, \beta_{m}\right)}{\left\|V_{(\mu)}\left(r, \beta_{m}\right)\right\|^{2}},
$$

$j=\overline{1, n+1}$

(4) Green's functions generated by the inhomogeneity of the conjugate conditions:

$$
R_{(\mu) ; i 2}^{j k}(t, r)=a_{k}^{2} \sigma_{k} \frac{\operatorname{sh} R_{k}}{c_{1 k}} \sum_{m=1}^{\infty} e^{-q_{m}^{2} t} Z_{(\mu) ; i 2}^{k}\left(\beta_{m}\right) \frac{V_{(\mu) ; j}\left(r, \beta_{m}\right)}{\left\|V_{(\mu)}\left(r, \beta_{m}\right)\right\|^{2}},
$$

$i=1,2, k=\overline{1, n}$.

As a result of application to the matrix-element $\left[\tilde{u}_{m}(t)\right]$, where a function $\tilde{u}_{m}(t)$ is defined by a formula (3.15), by rule of multiplication of matrices of operator matrixcolumn (3.7), we get the unique bounded solution of parabolic initial-boundary problem (3.8)-(3.11):

$$
\begin{aligned}
u_{j}(t, r)= & \sum_{k=1}^{n+1} \int_{0}^{t} \int_{R_{k-1}}^{R_{k}} H_{(\mu) ; j k}(t-\tau, r, \rho)\left[f_{k}(\tau, \rho)\right. \\
& \left.\quad+\delta_{+}(\tau) g_{k}(\rho)\right] \sigma_{k} \operatorname{sh} \rho d \rho d \tau \\
+ & \int_{-0}^{t}\left(W_{(\mu) ; 1 j}(t-\tau, r) g_{0}(\tau)+W_{(\mu) ; n+1, j}(t-\tau, r) g_{R}(\tau)\right) d \tau \\
+ & \sum_{k=1}^{n} \int_{0}^{t}\left(R_{(\mu) ; 12}^{j k}(t-\tau, r) \omega_{2 k}(\tau)\right. \\
& \left.\quad-R_{(\mu) ; 22}^{j k}(t-\tau, r) \omega_{1 k}(\tau)\right) d \tau,
\end{aligned}
$$

where $j=\overline{1, n+1}$, and $\delta_{+}(\tau)$ is the Dirac measure concentrated at $0^{+}$. The vectorfunction $u(t, r)=\left\{u_{1}(t, r), u_{2}(t, r), u_{3}(t, r), \ldots, u_{n}(t, r), u_{n+1}(t, r)\right\}$, the components $u_{i}(t, r)$ of which are determined by formulas (3.16), fully describes the exact analytical solution of the given parabolic problem. 
3.3. An example: a dynamics problem

Consider the problem to construct in the domain $D_{n}^{+}$the bounded solution of the separate hyperbolic system of equations with the generalized Legendres operator

$$
\left(\frac{\partial^{2}}{\partial t^{2}}+\chi_{j}^{2} u_{j}-a_{j}^{2} \Lambda_{(\mu) j}\right) u_{j}(t, r)=f_{j}(t, r), \quad j=\overline{1, n+1}
$$

under the initial conditions

$$
\left.u_{j}(t, r)\right|_{t=0}=\varphi_{j}(r),\left.\frac{\partial u_{j}}{\partial t}\right|_{t=0}=\psi_{j}(r), j=\overline{1, n+1},
$$

and the boundary conditions (3.10) and the conjugate conditions (3.11).

We assume that the compatibility conditions are satisfied:

$$
\begin{aligned}
\left.\left(\alpha_{11}^{0} \frac{d}{d r}+\beta_{11}^{0}\right) \varphi_{1}\right|_{r=R_{0}} & =g_{0}(0), \\
\left.\left(\alpha_{22}^{n+1} \frac{d}{d r}+\beta_{22}^{n+1}\right) \varphi_{n+1}\right|_{r=R_{n+1}} & =g_{R}(0) ; \\
{\left.\left[\left(\alpha_{j 1}^{k} \frac{d}{d r}+\beta_{j 1}^{k}\right) \varphi_{k}-\left(\alpha_{j 2}^{k} \frac{d}{d r}+\beta_{j 2}^{k}\right) \varphi_{k+1}\right]\right|_{r=R_{k}} } & =\omega_{j k}(0)
\end{aligned}
$$

for $j=1,2, k=\overline{1, n}$;

$$
\begin{array}{r}
\left.\left(\alpha_{11}^{0} \frac{d}{d r}+\beta_{11}^{0}\right) \psi_{1}\right|_{r=R_{0}}=g_{0}^{\prime}(0), \\
\left.\left(\alpha_{22}^{n+1} \frac{d}{d r}+\beta_{22}^{n+1}\right) \psi_{n+1}\right|_{r=R_{n+1}}=g_{R}^{\prime}(0),
\end{array}
$$

and

$$
\left.\left[\left(\alpha_{j 1}^{k} \frac{d}{d r}+\beta_{j 1}^{k}\right) \psi_{k}-\left(\alpha_{j 2}^{k} \frac{d}{d r}+\beta_{j 2}^{k}\right) \psi_{k+1}\right]\right|_{r=R_{k}}=\omega_{j k}^{\prime}(0)
$$

for $j=1,2, k=\overline{1, n}$. 
Constructed according to the logical scheme of solving parabolic problem (3.8)(3.11), the unique bounded solution of the given hyperbolic problem has the form

$$
\begin{aligned}
u_{j}(t, r)= & \sum_{k=1}^{n+1} \int_{0}^{t} \int_{R_{k-1}}^{R_{k}} H_{(\mu) ; j k}(t-\tau, r, \rho)\left(f_{k}(\tau, \rho)\right. \\
& \left.\quad+\delta_{+}(\tau) \psi_{k}(\rho)\right) \sigma_{k} \operatorname{sh} \rho d \rho d \tau \\
+ & \frac{\partial}{\partial t} \sum_{k=1}^{n+1} \int_{R_{k-1}}^{R_{k}} H_{(\mu) ; j k}(t, r, \rho) \varphi_{k}(\rho) \sigma_{k} \operatorname{sh} \rho d \rho \\
+ & \int_{0}^{t}\left(W_{(\mu) ; 1 j}(t-\tau, r) g_{0}(\tau)+W_{(\mu) ; n+1, j}(t-\tau, r) g_{R}(\tau)\right) d \tau \\
+ & \sum_{k=1}^{n} \int_{0}^{t}\left(R_{(\mu) ; 12}^{j k}(t-\tau, r) \omega_{2 k}(\tau)\right. \\
& \left.\quad-R_{(\mu) ; 22}^{j k}(t-\tau, r) \omega_{1 k}(\tau)\right) d \tau, \quad j=\overline{1, n+1} .
\end{aligned}
$$

The principal solutions appearing in formulas (3.17) are determined by the formulas given above, in which the function $\exp \left(-q_{m}^{2} t\right)$ is replaced by $q_{m}^{-1} \sin q_{m} t$. Note that the constructed solutions of statics, quasi-statics and dynamics problems are of algorithmic character and continuously rely on parameters and data of the problem under consideration. These solutions can be used in both theoretical research and engineering calculations.

\section{REFERENCES}

[1] N. A. Belova, "A certain integral expansion in spherical functions of the first and second kind," Differents. Uravn., vol. 5, pp. 2096-2100, 1969.

[2] N. Dunford and J. T. Schwartz, Linear Operators. Part I, ser. Wiley Classics Library. New York: John Wiley \& Sons Inc., 1988, General theory, With the assistance of William G. Bade and Robert G. Bartle, Reprint of the 1958 original, A Wiley-Interscience Publication.

[3] I. A. Fedotova, "On an integral transformation with generalized associated Legendre functions," Vychisl. Prikl. Mat. (Kiev), no. 71, pp. 33-43, 1990, transl. in J. Math. Sci., vol. 68, no. 6, pp. 759765, 1994.

[4] V. A. Fok, "On the expansion of an arbitrary function into the integral over Legendres functions with complex sign," C. R. Acad. Sci. URSS, vol. 39, no. 7, pp. 253-256, 1943.

[5] I. M. Konet, "On a generalized integral transform of Meler-Fok type on piece-homogeneity polar axis $r>r_{0}>0$," in International Scientific Conference Dedicated to the 60th Anniversary of the Chair of Integral and Differential Equations. Kiev: Kiev Shevchenko National University, 2005, p. 46, in Ukrainian.

[6] I. M. Konet, "On a generalized integral transform of Meler-Fok type on the polar axis with $n$ conjugate points," in Integral Equations and Their Applications, International Scientific Conference, Odessa, 2005, p. 70, in Ukrainian.

[7] I. M. Konet and M. P. Lenyuk, "A generalized integral transform of the Mehler-Fock type on the polar axis $r \geq R_{0}>3$ with $n$ contact points," Mat. Stud., vol. 25, no. 2, pp. 169-180, 2006. 
[8] I. M. Konet, M. P. Lenyuk, and O. M. Nikitina, "Some generalizations of integral transforms of Meler-Fok type,” Nats. Akad. Nauk Ukrain. Inst. Mat. Preprint, No. 98.6, Kiev, 1998, in Ukrainian.

[9] I. M. Konet and O. M. Nikitina, "The integral transform generated on the polar axis $r \geq 0$ by a generalized Legendres operator," in Modern Problems of Mathematics, International Scientific Conference, vol. 4. Chernivtsi: Ruta, 1998, pp. 47-50, in Ukrainian.

[10] I. M. Konet and O. M. Nikitina, "Integral transform of Meler-Fok type generated on the polar axis $r \geq r_{0}>0$ by a generalized Legendres differential operator," in Collection of Research Articles, ser. Physics and Mathematics. Kamyanets-Podilskii: Kamenets-Podilskii State University, 1998, no. 4, pp. 57-63, in Ukrainian.

[11] A. Kurosh, Higher Algebra. Moscow: Mir, 1988, translated from the Russian by George Yankovsky, Reprint of the 1972 translation.

[12] N. N. Lebedev, "Some integral transforms of mathematical physics," Ph.D. dissertation, Leningrad, 1951, in Russian.

[13] G. E. Shilov, Elementary Real and Complex Analysis. Mineola, NY: Dover Publications Inc., 1996, revised English edition translated from the Russian and edited by Richard A. Silverman.

[14] Y. S. Uflyand, Integral Transforms in Problems of Elasticity Theory, augmented ed. Leningrad: Nauka, 1967, in Russian.

[15] Y. S. Uflyand, "On some new integral transforms and their applications to problems of mathematical physics," in Questions of Mathematical Physics, Leningrad, 1976, pp. 93-106, in Russian.

[16] A. F. Ulitko, "On a generalization of the Meler-Fok integral transform," Prikl. Mekh., vol. 3, no. 5, pp. 45-49, 1967.

[17] N. A. Virchenko and I. A. Fedotova, Generalized Legendres Functions and Their Applications, Kiev, 1998, in Russian.

\section{Author's address}

\section{M. Konet}

Kamyanets-Podilsky State University, Department of Mathematics, 61 I. Ogiyenko St., 32300 Kamyanets-Podilsky, Ukraine 